\title{
Determinação da densidade de empacotamento de sistemas granulares compostos a partir da areia normal do IPT: comparação entre modelos de otimização de distribuição granulométrica e composições aleatórias
}

\section{(Determination of the particle packing of granular systems composed with the Brazilian standard sand from IPT: comparison between models for particle size distribution optimization and random compositions)}

\author{
C. Londero, L. A. Lenz, Í. M. R. dos Santos, N. S. Klein \\ Programa de Pós-Graduação em Engenharia de Construção Civil, Universidade Federal do Paraná, \\ ACF Centro Politécnico, Bloco III, Jardim das Américas, Curitiba, PR 81531-980 \\ carolinalondero7@gmail.com,lenzlauri@hotmail.com,icaro.santos@cimentoitambe.com.br,nayaraklein@ufpr.br
}

\begin{abstract}
Resumo
O empacotamento de partículas em concretos e argamassas adquire cada dia mais importância no âmbito técnico, uma vez que muitas das propriedades dos materiais compósitos são influenciadas pelo índice de vazios e concentração de sólidos. Este trabalho teve o objetivo de comparar a densidade de empacotamento de agregados miúdos, cujas curvas granulométricas foram obtidas pelo uso de diferentes modelos de empacotamento de partículas. A areia normal brasileira, fornecida pelo IPT, foi utilizada para verificação experimental dos resultados teóricos obtidos pelo uso dos modelos. As densidades de empacotamento das curvas granulométricas foram calculadas através do modelo de empacotamento CPM (do inglês, compressible packing model). A máxima densidade de empacotamento foi buscada pelo uso de modelos de empacotamento que resultam em uma curva granulométrica ideal, fazendo-se valer de ensaios preliminares que indicavam uma maior densidade de empacotamento na fração grossa da areia, de 1,2 mm. Desta forma, além das curvas granulométricas ideais, fez-se também um estudo com composições aleatórias onde houve aumento progressivo de 5\% na fração grossa da areia com a devida compensação nas frações mais finas. Utilizou-se, como referência, a curva granulométrica que representa a média dos limites inferior e superior da zona ótima recomendada pela norma NBR 7211. Os resultados obtidos mostraram que é possível aumentar a densidade de empacotamento através do uso de modelos de empacotamento de partículas e composições aleatórias, com relação à curva média da norma. Esse aumento foi da ordem de $2 \%$ e $5 \%$ para as densidades de empacotamento real e virtual, respectivamente. A verificação experimental dos resultados teóricos demonstrou que o modelo CPM infravalorou a densidade de empacotamento em aproximadamente $10 \%$.
\end{abstract}

Palavras-chave: empacotamento de partículas, modelos de empacotamento, granulometria, areia normal do IPT.

Abstract

The study of particle packing in concretes and mortars is becoming more and more important in the technical field, since the properties of the composite materials are influenced by both the voids index and the solid concentration. This paper aimed to compare the packing density of fine aggregates, whose grading curves were obtained by the use of different particle packing models. The Brazilian standard sand, provided by IPT, was used for the experimental validation of the theoretical results obtained by the use of the models. The packing densities of the grading curves were calculated with the compressible packing model (CPM). The maximum packing density was sought by using particle packing models resulting in an optimal particle size distribution curve. Preliminary tests were made, which indicated a higher packing density of the coarser fraction of the sand, $1.2 \mathrm{~mm}$. Therefore, in addition to the ideal grading curves, a study with random compositions was also made, with a progressive increase of $5 \%$ in the coarse sand fraction and a consequent decrease of the finer fractions. A grading curve representing the average of the lower and upper limits of the optimal zone recommended by the standard NBR 7211 was used as reference. The results showed that it is possible to increase the packing density using particle packing models and random compositions, with respect to the average grading curve from the Brazilian standard. An increase in the order of $2 \%$ and $5 \%$ was reached for the actual and the virtual packing densities, respectively. The experimental validation of the theoretical results showed that the CPM model undervalues the packing density in approximately $10 \%$.

Keywords: particle packing, packing models, particle size distribution, Brazilian standard sand from IPT.

\section{INTRODUÇÃO}

Os materiais compósitos empregados na construção civil são compostos por partículas de diferentes formas e tamanhos. Entre estes, podem-se citar os concretos e argamassas, formados por grãos de dimensões milimétricas, micrométricas e, em alguns casos, inclusive nanométricas. Além disso, os grãos podem apresentar forma bastante 
esférica, como é o caso da sílica ativa, ou grãos mais angulosos e, em alguns casos, alongados, como ocorre com alguns agregados de britagem. Toda essa variedade de grãos deve ser combinada de modo a ocupar o maior volume possível, para que o índice de vazios destes materiais seja sempre reduzido, favorecendo a resistência mecânica e durabilidade do material final. Em concretos e argamassas, a pasta de cimento preenche os espaços vazios entre os agregados, conectando esses grãos no estado endurecido, garantindo assim a integridade e propriedades mecânicas do material. Dentre os componentes do concreto, o cimento é o mais oneroso e resulta em maior impacto ao meio ambiente pela elevada emissão de $\mathrm{CO}_{2}$ durante seu processo de produção $[1,2]$. Uma forma de reduzir o consumo de cimento está atrelada a obter um esqueleto granular compacto, que apresente máxima densidade de empacotamento do agregado. Isso colabora para a redução do volume de pasta de cimento necessária para preencher os vazios existentes no esqueleto granular [3, 4]. Assim, para se obter esqueletos granulares compactos é necessário fazer o uso de modelos de empacotamento de partículas que permitam estabelecer a proporção e tamanho adequados dos agregados, de modo a obter um conjunto com um baixo índice de vazios. Diversos são os modelos existentes, cada qual considerando efeitos geométricos e de interação entre grãos, resultando em diferentes valores de densidades de empacotamento. Esta densidade é definida como o volume de sólidos em uma unidade de volume total e, estando associada ao índice de vazios, é de extrema relevância no estudo do empacotamento de agregados para a produção de concretos e argamassas [5-8].

A densidade de empacotamento é influenciada pelas características dos agregados, como distribuição granulométrica e morfologia das partículas [9]. Os agregados podem apresentar curvas granulométricas contínuas, descontínuas ou uniformes. Uma curva contínua, com grande amplitude de grãos, é considerada bem graduada quando existem frações pequenas em quantidade suficiente para preencher os espaços entre as partículas maiores, diminuindo ao máximo o volume de vazios e aumentando, assim, a densidade de empacotamento. Este efeito de preenchimento, conhecido como efeito fíler, resulta na diminuição da porosidade de um conjunto granular, aumentando a sua densidade de empacotamento $[10,11]$. A forma dos grãos, por sua vez, influencia na acomodação das partículas, uma em relação às outras. Quanto mais distante da forma esférica os grãos se apresentarem, maior será o espaço vazio entre as partículas, resultando na diminuição da densidade de empacotamento $[9,12]$.

O presente trabalho teve como objetivo realizar um estudo comparativo entre três modelos de empacotamento de partículas, buscando determinar qual dos modelos utilizados produz uma curva granulométrica com o menor índice de vazios. Foram estudados os modelos propostos por Füller e Thompson, Andreasen e Andersen, ambos descritos por [13], e Funk e Dinger [14]. Além disso, sete curvas granulométricas foram compostas a partir de uma curva de referência, sendo esta a curva granulométrica que representa a média dos limites inferior e superior da zona ótima recomendada pela norma NBR 7211 [15]. Dentre estas sete composições aleatórias, buscou-se determinar também a que apresentasse o menor índice de vazios. No total, foram estudadas onze curvas granulométricas. $\mathrm{O}$ índice de vazios destas curvas foi calculado segundo o modelo CPM (do inglês, compressible packing model) de De Larrard [16]. A verificação experimental dos dados teóricos obtidos foi realizada para três composições granulométricas (a curva média da norma, tida como referência, e as curvas que apresentaram a máxima densidade de empacotamento dentre os modelos e as composições aleatórias estudadas). Para isso, utilizou-se a areia normal brasileira em suas quatro frações disponíveis, sendo estas $1,2,0,6,0,3$ e 0,15 $\mathrm{mm}$, conforme preconiza a norma NBR 7214 [17].

\section{Modelos de empacotamento de partículas}

Desde o início do século XX diversos pesquisadores estudaram o efeito da distribuição granulométrica dos agregados sobre o empacotamento de partículas, buscando encontrar uma curva granulométrica ideal para uso em concretos. Os pioneiros neste desafio foram Füller e Thompson, em 1907, que propuseram uma curva granulométrica de distribuição contínua, dada pela Equação $\mathrm{A}$, para situações em que o expoente $q$ é igual a 0,5 . Esta mesma equação foi proposta por Andreasen e Andersen em 1930, porém, estes autores não especificaram um valor para o expoente $q$, de modo que diferentes valores poderiam ser usados, resultando em variações nas curvas granulométricas obtidas [7, 13]. Mais tarde, simulações computacionais demonstraram que, quando o coeficiente $q$ assume o valor de 0,37 , é possível obter a máxima densidade de empacotamento do conjunto granular [14]. É importante ressaltar que estes modelos não consideram a forma das partículas na determinação da curva granulométrica ideal, apenas seu diâmetro. Além disso, a Equação A não tem um limite mínimo de tamanho de partículas, de modo que se admite partículas infinitamente pequenas, o que não condiz com uma situação real. Outras equações foram desenvolvidas ao longo do tempo para expressar a distribuição granulométrica ideal dos agregados. Esta necessidade foi observada por Funk e Dinger [14] que, a partir do modelo de Andreasen e Andersen, adicionaram um limite inferior ao conjunto de partículas, como se pode observar através da Equação B.

$$
\begin{aligned}
& \operatorname{CPFT}(\%)=100 \cdot\left(\frac{\mathrm{D}}{\mathrm{D}_{\max }}\right)^{\mathrm{q}} \\
& \operatorname{CPFT}(\%)=100 \cdot\left(\frac{\mathrm{D}^{\mathrm{q}}-\mathrm{Dmin}^{\mathrm{q}}}{\mathrm{Dmax}^{\mathrm{q}}-\mathrm{Dmin}^{\mathrm{q}}}\right)
\end{aligned}
$$

em que $C P F T(\%)$ é a porcentagem volumétrica de partículas menores que o diâmetro $D$ (do inglês cumulative percent finer than $D), D_{\min }$ é o diâmetro da menor partícula, $D$ é diâmetro da partícula, $D_{\max }$ corresponde ao o diâmetro da maior 
partícula do conjunto e $q$ é o coeficiente de distribuição.

Além de modelos que buscam produzir curvas granulométricas ideais, foram difundidos vários modelos analíticos, cujas equações permitem determinar a concentração de sólidos e, consequentemente, o índice de vazios dos conjuntos granulares. Esses modelos consideram desde combinações binárias [18, 19] a várias classes de tamanhos de partículas em um mesmo conjunto [13, 16, 20, 21]. Dentre os modelos analíticos, o modelo de De Larrard [16] está dentre os mais conhecidos, tendo sido escolhido para ser usado no presente estudo pela coerência matemática apreciada na dedução das equações propostas. O modelo de De Larrard [16], denominado CPM, permite prever a densidade de empacotamento de conjuntos granulares polidispersos compostos por $n$ classes de tamanhos de grãos. $\mathrm{O}$ modelo demanda como dado de entrada a densidade de empacotamento de cada uma das classes componentes do conjunto. Para agregados, esse dado pode ser obtido a partir da determinação da massa unitária e do índice de vazios, segundo a norma NBR NM 45 [22].

$\mathrm{O}$ modelo CPM considera os efeitos de interação entre as partículas que ocorrem quando há proximidade entre grãos. Quando o diâmetro de uma determinada classe de partículas for maior que a dimensão dos vazios deixados pelo arranjo da classe com diâmetro imediatamente superior, ocorre um afastamento dos grãos maiores, caracterizando o efeito de afastamento (Fig. 1). Nesta figura observa-se também o efeito parede, que ocorre quando os diâmetros das partículas de duas classes são tão diferentes que a superfície de contato do grão mais grosso funciona como um plano ao contato com os grãos mais finos, aumentando os vazios na região próxima à superfície. Os coeficientes $a_{i j}$ e $b_{i j}$ representam o efeito de afastamento e o efeito parede, respectivamente, e são determinados através das Equações C e D. O cálculo destes coeficientes compara sempre duas classes de tamanhos de grãos, devendo ser realizadas todas as combinações possíveis entre as $n$ classes que compõem o conjunto. A classe dominante é chamada de classe $i$,

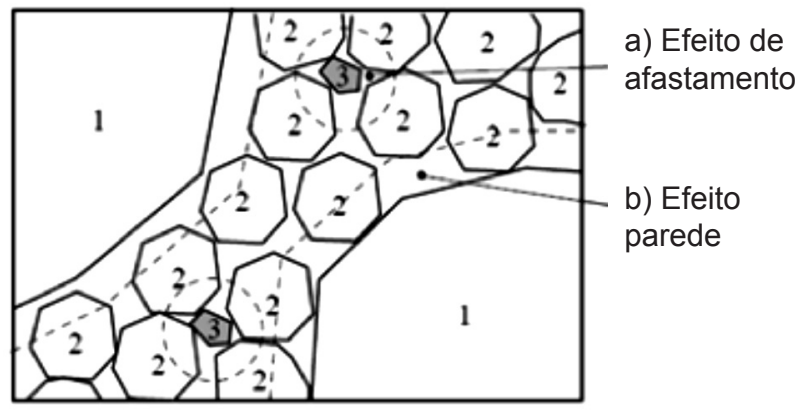

Figura 1: Interação entre partículas: a) efeito de afastamento nos grãos de classe 2 provocado pela classe 3 de grãos; e b) efeito parede, em que grãos da classe 2 são influenciados pela proximidade com grãos de classe 1 [16].

[Figure 1: Particle interaction: a) loosening effect in grains of class 2 exerted by grains of class 3; and b) wall effect, in which grains of class 2 are influenced by the proximity with grains of class 1 [16].] sendo $d_{i}$ o diâmetro de suas partículas. Em um conjunto granular composto por quatro classes, supondo a classe $n=1$ dominante, esta é chamada de classe $i$, sendo $i=1$. Todas as demais classes $(n=2, n=3$ e $n=4)$ são chamadas de classe $j$, sendo $j=2, j=3$ e $j=4$. Desse modo, as combinações possíveis entre as quatro classes resultam no cálculo dos coeficientes $a_{12}, a_{13}$ e $a_{14}$ para determinar o efeito de afastamento e $b_{12}, b_{13}$ e $b_{14}$ para determinar o efeito parede. Em uma segunda etapa de cálculo, há que se considerar a classe $n=2$ dominante (i), sendo as demais $(n=1, n=3$ e $n=4)$ tidas como complementares $(j)$, calculando novamente os coeficientes $a_{i j}$ e $b_{i j}$, e assim por diante, até que todas as classes de grãos sejam consideradas como dominantes [16].

$$
\begin{aligned}
& \mathrm{a}_{\mathrm{ij}}=\sqrt{1-\left(1-\mathrm{d}_{\mathrm{j}} / \mathrm{d}_{\mathrm{i}}\right)^{1,02}} \\
& \mathrm{~b}_{\mathrm{ij}}=1-\left(1-\mathrm{d}_{\mathrm{j}} / \mathrm{d}_{\mathrm{i}}\right)^{1,50}
\end{aligned}
$$

Estes coeficientes são considerados no cálculo da densidade de empacotamento virtual para um conjunto composto por $n$ classes, segundo mostrado nas Equações $\mathrm{E}$ e F, sendo necessário determinar previamente a densidade de empacotamento de cada classe $\left(\beta_{i}\right)$ de forma experimental e estabelecer o volume complementar de cada classe do conjunto $\left(y_{i}\right)$. A densidade de empacotamento virtual do conjunto granular é definida como a densidade de empacotamento encontrada quando cada partícula do sistema mantém sua forma original sendo posicionada uma a uma, o que não é viável de se realizar na prática. Além disso, ressalta-se a necessidade de calcular a densidade de empacotamento virtual considerando cada uma das classes como dominante $\left(\gamma_{i}\right)$, sendo o valor mínimo obtido nas $n$ situações consideradas igual à densidade de empacotamento virtual do conjunto $(\gamma)[16]$ :

$$
\begin{gathered}
\gamma_{i}=\frac{\beta_{i}}{1-\sum_{j-1}^{i-1}\left[1-\beta_{i}+b_{i j} \cdot \beta_{i}\left(1-\frac{1}{\beta_{j}}\right)\right] \cdot y_{j}-\sum_{j=i+1}^{n}\left[1-\alpha_{i j} \cdot \frac{\beta_{i}}{\beta_{j}} \cdot y_{j}\right.} \\
\gamma=\operatorname{MIN}\left(\gamma_{i}\right)(1 \leq \mathrm{i} \leq n)
\end{gathered}
$$

Para se determinar o valor da densidade de empacotamento real do conjunto granular $(\phi)$, considera-se a compactação dos grãos através do índice de compactação $(K)$. Desse modo, a densidade de empacotamento real pode ser calculada pela Equação G:

$$
\mathrm{K}=\sum_{\mathrm{i}=1}^{\mathrm{n}} \frac{\mathrm{y}_{\mathrm{i}} / \beta_{\mathrm{i}}}{1 / \phi-1 / \mathrm{y}_{\mathrm{i}}}
$$

A Tabela I apresenta diferentes valores do índice de compactação para diversos tipos de energia de compactação, tendo sido estes determinados experimentalmente por [16]. Para um melhor entendimento do uso das equações apresentadas pelo modelo CPM, no Apêndice apresenta-se uma rotina de cálculo considerando o exemplo de um dos conjuntos granulares estudados no presente artigo, sendo 
Tabela I - Valores do índice de compactação K [16]. [Table I - Compaction index K [16].]

\begin{tabular}{cc}
\hline Processo de compactação & $\mathrm{K}$ \\
\hline Lançamento simples & 4,1 \\
Apiloamento & 4,5 \\
Vibração & 4,75 \\
Demanda de água & 6,7 \\
Vibração e compressão de $10 \mathrm{kPa}$ & 9 \\
Empacotamento virtual & $\infty$ \\
\hline
\end{tabular}

este o conjunto granular 4 (descrito no item seguinte).

\section{EXPERIMENTAL}

\section{Materiais}

A areia normal brasileira, fornecida pelo IPT, foi utilizada como agregado miúdo no presente trabalho. Essa areia é empregada como material de referência para a realização de ensaios físicos e mecânicos de cimento Portland e está disponível em quatro frações: areia grossa $(1,2 \mathrm{~mm})$, média grossa $(0,6 \mathrm{~mm})$, média fina $(0,3 \mathrm{~mm})$ e fina $(0,15 \mathrm{~mm})$. A areia normal utilizada segue as especificações estabelecidas na norma NBR 7214 [17]. Sua massa específica $\left(\gamma_{a}\right)$ é igual a $2618 \mathrm{~kg} / \mathrm{m}^{3}$, tendo sido determinada segundo a norma NBR NM 53 [23]. A massa unitária de cada classe $\left(\rho_{i}\right)$, determinada pelo método A (massa unitária compactada de agregado com diâmetro máximo inferior ou igual a 37,5 $\mathrm{mm}$ ) descrito na norma NBR NM 45 [22], assim como a densidade de empacotamento $\left(\beta_{i}\right)$ de cada uma das quatro classes de tamanhos de grãos, encontra-se apresentada na Tabela II. Determinou-se a massa unitária de cada classe três vezes, obtendo como valor final a média dos resultados. A densidade de empacotamento das classes foi determinada segundo a Equação H:

$$
\beta_{\mathrm{i}}=1-\frac{\mathrm{y}_{\mathrm{a}}-\rho_{\mathrm{i}}}{\mathrm{y}_{\mathrm{a}}}
$$

Os resultados apresentados na Tabela II demonstram que, para esse material em estudo, quanto maior o tamanho dos grãos que compõem a classe, maior a massa unitária e a densidade de empacotamento encontrada. Isso se deve ao arranjo espacial das partículas da areia normal do IPT no conjunto. Além disso, quanto mais finos os grãos, menor é a influência da gravidade, de modo que a interação entre partículas se torna cada vez mais importante [13, 24], podendo colaborar para o aumento da quantidade de vazios se aglomerados forem formados. Em se tratando de uma areia, é possível que aglomerados se formem na classe de menor tamanho, igual a $0,15 \mathrm{~mm}$. Observa-se também que as densidades de empacotamento obtidas para cada classe apresentam valores sempre menores que 0,450 . Este valor pode ser considerado baixo, sabendo-se que a máxima densidade de empacotamento que se atinge em misturas com grãos uniformes é igual a 0,740 , conforme proposto pelo matemático alemão Johannes Kepler no século XVII, problema este comprovado numericamente apenas no início do século XXI [25]. Esta densidade de empacotamento máxima é alcançada considerando o empacotamento de uma rede cúbica de face centrada de partículas perfeitamente esféricas, onde cada partícula foi posicionada no sistema uma a uma. Desse modo, quando se tem um conjunto granular formado a partir de condições diferentes das ideais apontadas, como é o caso da areia estudada, os valores encontrados são naturalmente menores que o valor máximo.

\section{Método}

Buscando encontrar a composição granulométrica que apresentasse a maior densidade de empacotamento, entre as quatro frações da areia normal do IPT, os modelos de Füller e Thompson, Andreasen e Andersen, descritos por [13], e Funk e Dinger [14] foram utilizados. Para o cálculo segundo Füller-Thompson utilizou-se um coeficiente de distribuição $q$ igual a 0,5. Já para Andreasen-Andersen e Funk-Dinger, adotou-se $q$ igual a 0,37. Elaborou-se também uma curva média dos limites inferior e superior da zona ótima recomendada pela norma NBR 7211 [15]. Como a norma brasileira considera porcentagens retidas das frações 4,8 e $2,4 \mathrm{~mm}$, as quais não são disponibilizadas pela areia normal do IPT, optou-se por considerar que não houve material retido nessas frações possibilitando a comparação de resultados. A partir das curvas granulométricas resultantes dos modelos e da densidade de empacotamento das classes individuais da areia, dados na Tabela II, calculou-se a densidade de empacotamento dos conjuntos referentes a cada curva granulométrica construída, utilizando para isto o modelo analítico CPM [16]. O índice de compactação $K$ utilizado no cálculo da densidade de empacotamento real foi igual a 4,5 , correspondente ao processo de compactação por

Tabela II - Massa unitária e densidade de empacotamento das classes da areia. [Table II - Bulk density and packing density of the sand classes.]

\begin{tabular}{lcccc}
\hline \multirow{2}{*}{ Propriedade } & \multicolumn{4}{c}{ Classe $(\mathrm{mm})$} \\
& 1,2 & 0,6 & 0,3 & 0,15 \\
\hline Massa unitária compactada $\left(\mathrm{kg} / \mathrm{m}^{3}\right)$ & 1155 & 1127 & 1110 & 1100 \\
Desvio padrão $\left(\mathrm{kg} / \mathrm{m}^{3}\right)$ & 1,22 & 1,27 & 1,42 & 1,94 \\
Densidade de empacotamento, $\beta_{\mathrm{i}}$ & 0,441 & 0,430 & 0,424 & 0,420 \\
\hline
\end{tabular}


Tabela III - Combinações volumétricas entre as 4 classes da areia normal brasileira.

[Table III - Volumetric combination between the 4 classes of the Brazilian standard sand.]

\begin{tabular}{ccccc}
\hline $\begin{array}{c}\text { Conjunto } \\
\text { granular }\end{array}$ & \multicolumn{4}{c}{ Volume de cada fração (\%) } \\
\hline 1 & $25,2 \mathrm{~mm}$ & $0,6 \mathrm{~mm}$ & $0,3 \mathrm{~mm}$ & $0,15 \mathrm{~mm}$ \\
2 & 30,0 & 25,0 & 25,0 & 25,0 \\
3 & 35,0 & 25,0 & 20,0 & 20,0 \\
4 & 40,0 & 20,0 & 20,0 & 20,0 \\
5 & 45,0 & 20,0 & 20,0 & 15,0 \\
6 & 50,0 & 20,0 & 15,0 & 15,0 \\
7 & 55,0 & 15,0 & 15,0 & 15,0 \\
\hline
\end{tabular}

apiloamento, conforme indicado previamente na Tabela I.

Para complementar o estudo, foram estabelecidas sete combinações volumétricas entre as classes da areia normal brasileira, sem que estas combinações obedecessem a qualquer modelo de empacotamento de partículas. Estas combinações são apresentadas na Tabela III. Iniciou-se com um volume de agregado igual para cada uma das 4 frações estudadas. Na sequência, foi-se aumentando $5 \%$ do volume na fração grossa, de 1,2 mm, a qual apresentou a maior densidade de empacotamento entre as 4 classes estudadas. Para as outras frações, foi-se diminuindo $5 \%$ do volume de agregados, iniciando as reduções sempre da fração mais fina para a mais grossa. Cada combinação adotada gerou uma curva granulométrica distinta, tendo sido determinada a densidade de empacotamento de cada conjunto também pelo modelo CPM [16].

Por fim, dentre as curvas granulométricas obtidas pelos modelos de empacotamento, foi escolhida a de maior densidade de empacotamento para a reprodução laboratorial a fim de analisar, comparativamente, os resultados teóricos (densidade de empacotamento virtual e real) com o resultado experimental. $\mathrm{O}$ mesmo foi realizado para as combinações aleatórias. A curva média dos limites da zona ótima da norma NBR 7211 [15], utilizada como referência, também foi submetida a ensaio laboratorial. Logo, foram verificados experimentalmente os resultados de três dentre as onze composições granulométricas estudadas. Para isso, realizou-se o ensaio de massa unitária pelo método A (massa unitária compactada de agregado com diâmetro máximo inferior ou igual a $37,5 \mathrm{~mm}$ ) descrito na norma NBR NM 45 [22]. A partir deste, foi possível determinar a densidade de empacotamento experimental da mistura, através da Equação $\mathrm{H}$, possibilitando a comparação dos resultados experimentais com os valores obtidos teoricamente pelo uso do modelo analítico.

\section{RESULTADOS E DISCUSSÃO}

\section{Curvas granulométricas}

Os resultados obtidos pela aplicação dos modelos de empacotamento de partículas, assim como a curva média da zona ótima estabelecida pela norma NBR 7211 [15], são dados na Tabela IV. Foi utilizado um diâmetro máximo de 2,4 mm para todos os modelos, pois ao calcular o volume de cada fração, os modelos consideram sempre que o diâmetro máximo não apresenta porcentagem retida, excluindo da composição estes grãos. Caso não fosse utilizado o diâmetro máximo de 2,4 mm, a fração de 1,2 mm iria apresentar uma porcentagem de partículas igual a zero, o que impossibilitaria utilizar as quatro frações da areia normal do IPT. Os modelos de Füller-Thompson e AndreasenAndersen admitem partículas infinitamente pequenas, pois não consideram a existência de um diâmetro mínimo (Equação A). Este fato difere da realidade dos conjuntos granulares. Dessa forma, ao aplicar-se estes modelos para obtenção de uma curva granulométrica ideal, observa-se que a soma das porcentagens correspondentes a cada classe da areia não totaliza 100\% (Tabela IV). Este é um requisito indispensável para a avaliação de um conjunto granular real e a aplicação do modelo analítico CPM [16] para o cálculo da densidade de empacotamento. Pelo fato de que a soma

Tabela IV - Combinações volumétricas obtidas pelo uso dos modelos de empacotamento (os valores apresentados entre parêntesis são referentes à normalização dos resultados dados pelos modelos).

[Table IV - Volumetric combination obtained by the use of the packing models (the values given between parentheses refer to the normalization of the results given by the models).]

\begin{tabular}{lccccc}
\hline \multirow{2}{*}{ Conjunto granular } & \multicolumn{4}{c}{ Volume de cada fração (\%) } & \multirow{2}{*}{ Total (\%) } \\
\cline { 2 - 5 } & $1,2 \mathrm{~mm}$ & $0,6 \mathrm{~mm}$ & $0,3 \mathrm{~mm}$ & $0,15 \mathrm{~mm}$ & \\
\hline \multirow{2}{*}{ Modelo Füller e Thompson } & 29,29 & 20,71 & 14,64 & 10,36 & 75,0 \\
\multirow{2}{*}{ Modelo Andreasen e Andersen } & $(39,05)$ & $(27,61)$ & $(19,52)$ & $(13,82)$ & $(100,0)$ \\
Modelo Funk e Dinger & 22,62 & 17,50 & 13,54 & 10,48 & 64,14 \\
\multirow{2}{*}{ Média da zona ótima da norma NBR 7211 [15] } & $(35,26)$ & $(27,29)$ & $(21,11)$ & $(16,34)$ & $(100,0)$ \\
\hline
\end{tabular}



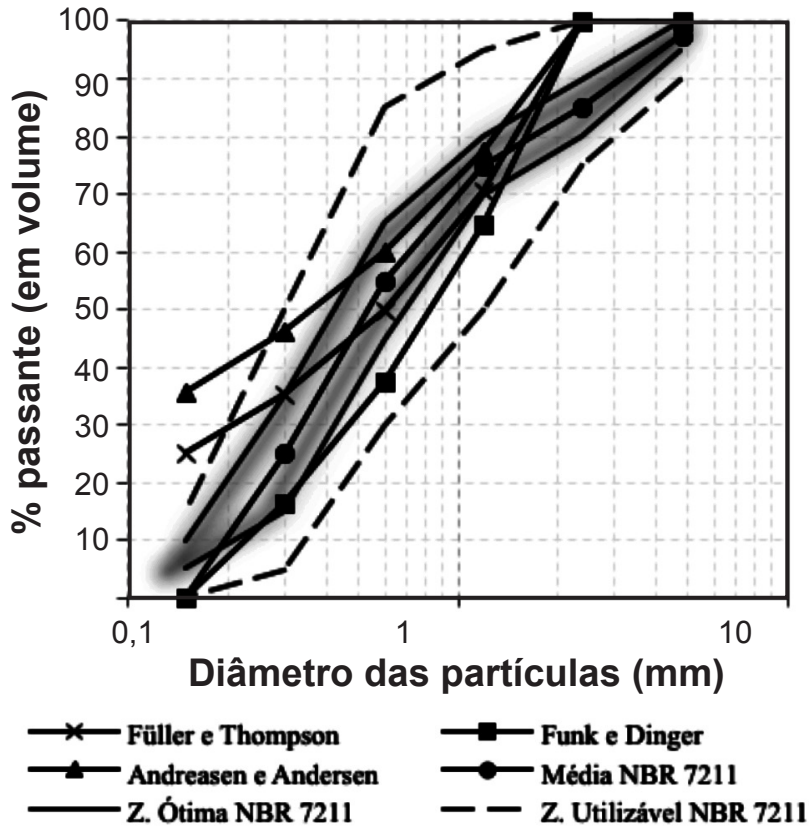

Figura 2: Curvas granulométricas obtidas pelos modelos e curva média da zona ótima estabelecida pela NBR 7211 [15].

[Figure 2: Grading curves obtained by the models and curve for the optimal zone established by the NBR 7211 [15].]

das porcentagens correspondentes a cada classe da areia não totalizar $100 \%$ nas curvas de Füller-Thompson e AndreasenAndersen (Tabela IV), optou-se por realizar a normalização dos resultados, os quais estão descritos entre parênteses na referida tabela. Para isso dividiram-se os valores percentuais de cada fração pela soma total atingida. Isso possibilitou o cálculo da densidade de empacotamento, através do método de CPM [16]. A curva normalizada determinada pelo modelo de Andreasen-Andersen apresentou os mesmos resultados da curva de Funk-Dinger. Isso ocorreu, pois ao aplicar a técnica de normalização elimina-se a possibilidade de haver grãos infinitamente pequenos, o que é semelhante a considerar um tamanho mínimo de grãos. Este último conceito foi implementado por Funk e Dinger [14] ao desenvolverem seu modelo (Equação B). Naturalmente, este fato faz com que as densidades de empacotamento destes dois conjuntos granulares sejam iguais, conforme se apresenta mais adiante.

A Fig. 2 apresenta as curvas granulométricas obtidas a partir das porcentagens volumétricas calculadas pelos modelos de empacotamento de partículas, além da curva obtida a partir da média dos limites da zona ótima na norma NBR 7211, dadas na Tabela IV. Semelhantemente, a Fig. 3 mostra as curvas correspondentes às combinações volumétricas aleatórias apresentadas previamente na Tabela III.Apartir da Fig. 2, observa-se que as curvas granulométricas referentes aos modelos de Füller-Thompson e AndreasenAndersen apresentaram comportamento semelhante, pois ambas não alcançaram o valor de ordenada zero, devido à não consideração de um diâmetro mínimo (Equação A). Observou-se que a primeira curva, de Füller-Thompson, foi mais próxima da curva utilizada como referência, obtida a partir da média dos percentuais que representam a zona
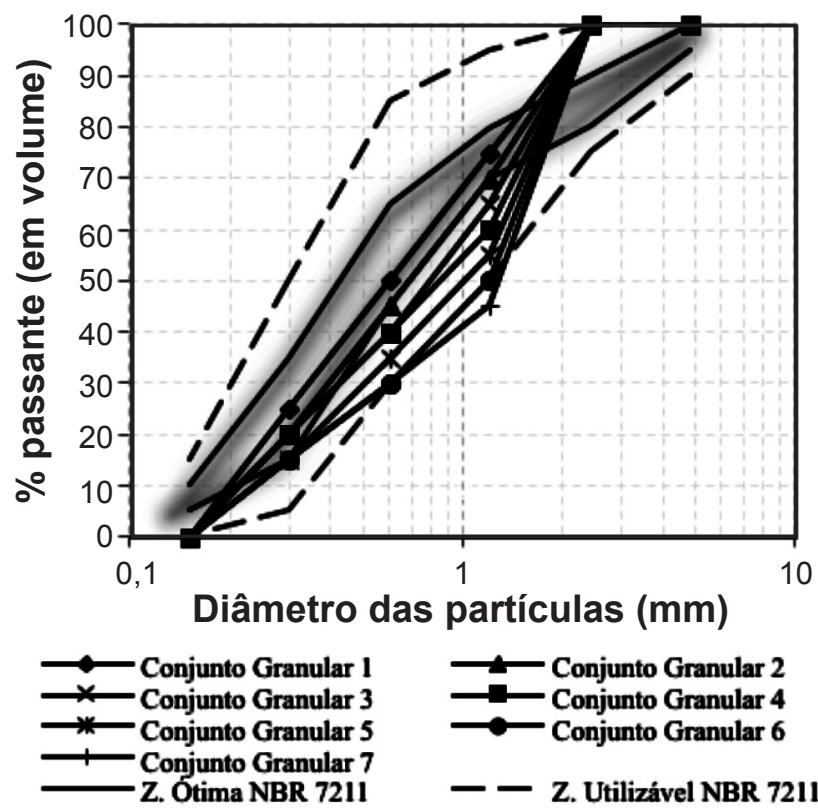

Figura 3: Curvas granulométricas obtidas pelas combinações desenvolvidas, segundo descrito na Tabela III.

[Figure 3: Grading curves obtained by the developed combinations, according to Table III.]

ótima da norma NBR 7211 [15]. Já a curva de Funk-Dinger se distanciou da zona ótima proposta pela mesma norma, passando à zona utilizável inferior. $\mathrm{O}$ mesmo aconteceu na Fig. 3, à medida que se diminuiu a porcentagem volumétrica das classes mais finas da areia; ao passar-se do conjunto granular 1 ao 7, suas respectivas curvas granulométricas se distanciaram da curva granulométrica que representa a média entre os limites da zona ótima da norma brasileira.

\section{Densidade de empacotamento dos conjuntos granulares}

Com as porcentagens volumétricas referentes a cada classe de tamanho de grãos e com os dados apresentados na Tabela II, aplicou-se o modelo analítico CPM [16] a fim de se obter a densidade de empacotamento de cada um dos conjuntos granulares propostos nas Fig. 2 e 3 . Os resultados obtidos a partir deste modelo são apresentados na Fig. 4. Observou-se que o conjunto granular 4 foi o que resultou na maior densidade de empacotamento, a qual atingiu 0,565 para a densidade de empacotamento virtual e 0,451 para a densidade de empacotamento real. Esse conjunto granular apresentou $40 \%$ do material retido na fração mais grossa, de $1,2 \mathrm{~mm}$, e os outros $60 \%$ do volume do material dividido igualmente entre as frações menores, de 0,6, 0,3 e 0,15 $\mathrm{mm}$. A curva granulométrica correspondente se encontrou dentro dos limites de utilização inferior da norma NBR 7211 [15]. Essa distribuição igualitária entre as frações finas permitiu um preenchimento uniforme dos vazios existentes, resultando em uma densidade de empacotamento mais elevada. É interessante observar que a curva média da zona ótima estabelecida pela norma NBR 7211 [15] foi a que obteve a menor densidade de empacotamento virtual, 


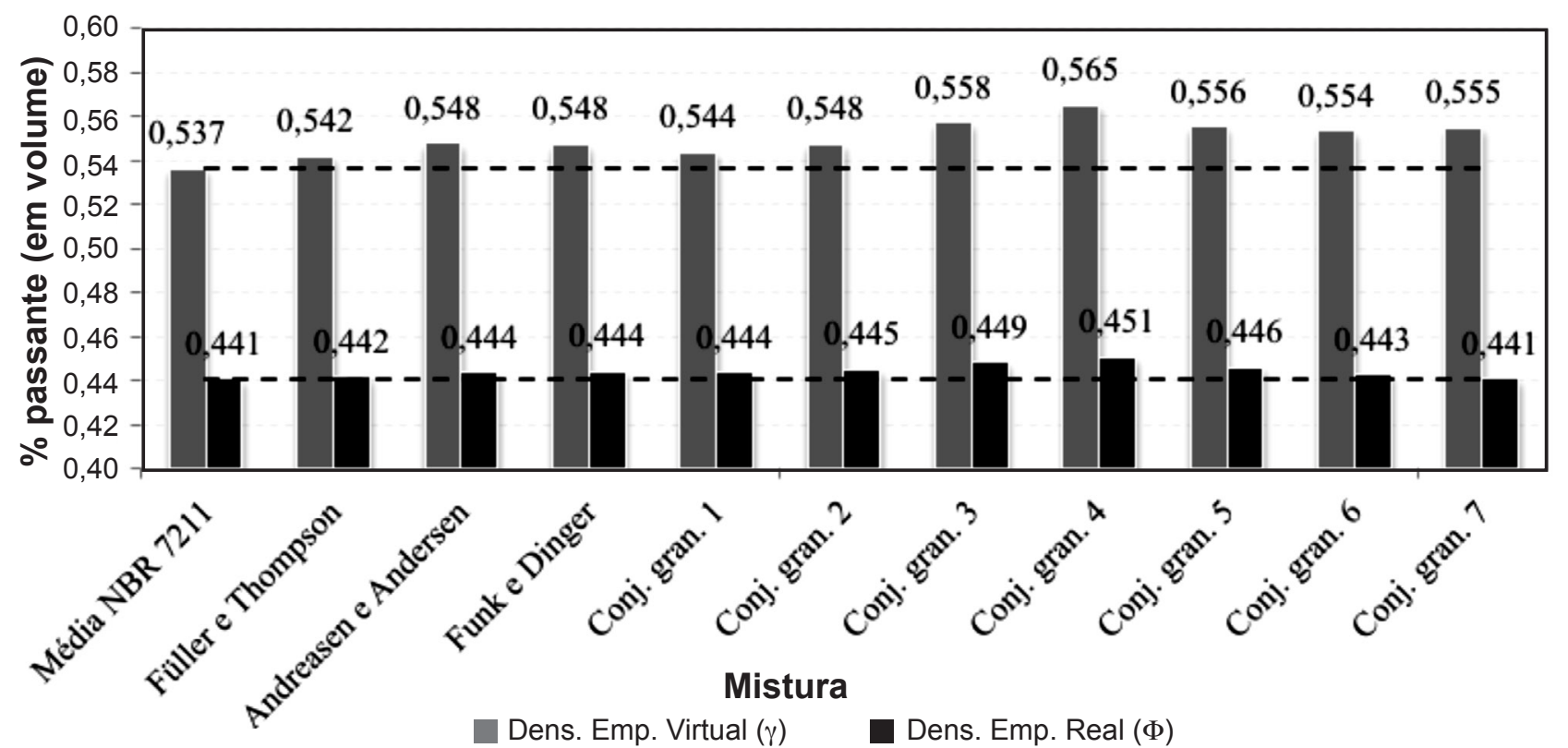

Figura 4: Densidades de empacotamento dos conjuntos granulares, calculadas segundo o modelo CPM [16].

[Figure 4: Packing density of the granular assemblies, calculated using the CPM model [16].]

igual a 0,537 , e real, igual a 0,441 , dentre todos os conjuntos granulares estudados. A densidade de empacotamento virtual obtida para esta curva, estabelecida como curva de referência foi $5,2 \%$ inferior à curva que obteve a maior densidade de empacotamento (conjunto granular 4). Se for considerada a densidade de empacotamento real, esta diferença cai para $2,2 \%$. Todos os demais conjuntos granulares estudados apresentaram resultados de densidade de empacotamento intermediários aos valores apresentados pela curva de referência (curva média da norma) e a curva do conjunto granular 4 , sendo estas diferenças pequenas, inferiores a $5,2 \%$ e $2,2 \%$ para as densidades de empacotamento virtual e real, respectivamente.

A curva granulométrica obtida pelo modelo de FüllerThompson, em que se fez a normalização dos dados, foi a que apresentou mais similaridade com a curva de referência da norma, resultando em densidades de empacotamento virtual e real $0,9 \%$ e $0,2 \%$ superiores, respectivamente. Já a curva granulométrica estabelecida através do modelo de empacotamento proposto por Funk e Dinger [14] apresentou densidades de empacotamento virtual e real $2,0 \%$ e $0,7 \%$ superiores aos valores obtidos pela curva de referência da norma, respectivamente. Semelhantemente, essa mesma curva do modelo resultou em densidades de empacotamento virtual e real 3\% e 1,6\% inferiores aos valores obtidos pelo conjunto granular 4 , de maior compacidade dentre os estudados. Os resultados de densidade de empacotamento obtidos pelos modelos de Andreasen-Andersen e Funk-Dinger foram iguais, pois a normalização dos resultados do primeiro modelo geram valores idênticos aos apresentados pelo segundo, conforme já comentado anteriormente. É importante ressaltar que, dentre os modelos de empacotamento estudados, o de Funk-Dinger foi o que apresentou maiores resultados de densidade de empacotamento. Da mesma forma, dentre as composições aleatórias estudadas, o conjunto granular 4 foi o que apresentou as maiores densidades de empacotamento. É possível notar, então, através das Figs. 2 e 3, que ambas as curvas granulométricas de Funk-Dinger e do conjunto granular 4 encontraram-se localizadas na zona utilizável inferior proposta pela norma NBR 7211 [15]. Esse fato demonstrou que uma curva granulométrica presente nesta região, zona utilizável inferior, é mais efetiva em termos de ocupação de espaço pelos grãos, quando comparada a uma curva granulométrica localizada inteiramente na zona ótima proposta pela mesma norma.

De qualquer forma, essas pequenas variações encontradas entre os resultados obtidos para os diversos conjuntos granulares, assim como os baixos valores de densidades reais de empacotamento encontrados (todos inferiores a 0,500), pode responder ao fato de que o presente estudo contou com o uso de apenas 4 classes de tamanho de grãos, dando uma amplitude pequena às curvas granulométricas estudadas, de 0,15 a 1,2 mm. Porém, a norma brasileira NBR 7211 [15] considera a existência de 6 classes de grãos para a areia, as quais não foram todas consideradas neste estudo devido à areia normal do IPT não dispor de outras frações. Além disso, os modelos de empacotamento de partículas que resultam em uma curva granulométrica ideal, como o de Funk e Dinger [14], devem ser usados considerando todo o esqueleto granular que será aplicado nos concretos, o que inclui as classes de tamanhos de grãos referentes à areia e à brita. Esse fato colabora ainda mais para aumentar a amplitude de tamanhos de grãos presentes nos conjuntos granulares. Uma mistura que dispõe de um maior número de classes pode apresentar um melhor preenchimento dos vazios, uma vez que há mais variedade de tamanhos de grãos, conforme já apontado por outros autores [13, 26]. 
De qualquer forma, são necessários estudos futuros que relacionem a quantidade de classes com a densidade de empacotamento para quantificar tal efetividade.

\section{Verificação experimental dos resultados teóricos}

Na Fig. 5 são apresentados os resultados de densidade de empacotamento determinados experimentalmente, conforme o método A da NBR NM 45 [22] (massa unitária compactada de agregado com diâmetro máximo inferior ou igual a $37,5 \mathrm{~mm}$ ) e Equação $\mathrm{H}$, em comparação aos resultados teóricos de densidade de empacotamento virtual e real, calculada pelo modelo CPM [16]. Como a densidade de empacotamento real é calculada utilizando o índice de compactação $\mathrm{K}$, correspondente ao método de compactação utilizado na determinação experimental, a comparação destes resultados experimentais deve ser realizada com os valores de densidade de empacotamento real. A comparação de dados experimentais não pode ser feita com a densidade de empacotamento virtual, pois esta não considera os efeitos de compactação que existem na prática. Ressalta-se que foram reproduzidos experimentalmente três conjuntos granulares: a curva da média dos valores recomendados para a zona ótima da norma NBR 7211 [15], sendo esta curva de referência, o conjunto granular dado pelo modelo de Funk e Dinger [14] e o conjunto granular 4, cujas curvas granulométricas apresentaram a máxima densidade de empacotamento dentre os modelos e os conjuntos aleatórios estudados, respectivamente.

Ao analisar os resultados apresentados na Fig. 5, percebese que densidade de empacotamento experimental foi $11,6 \%$,

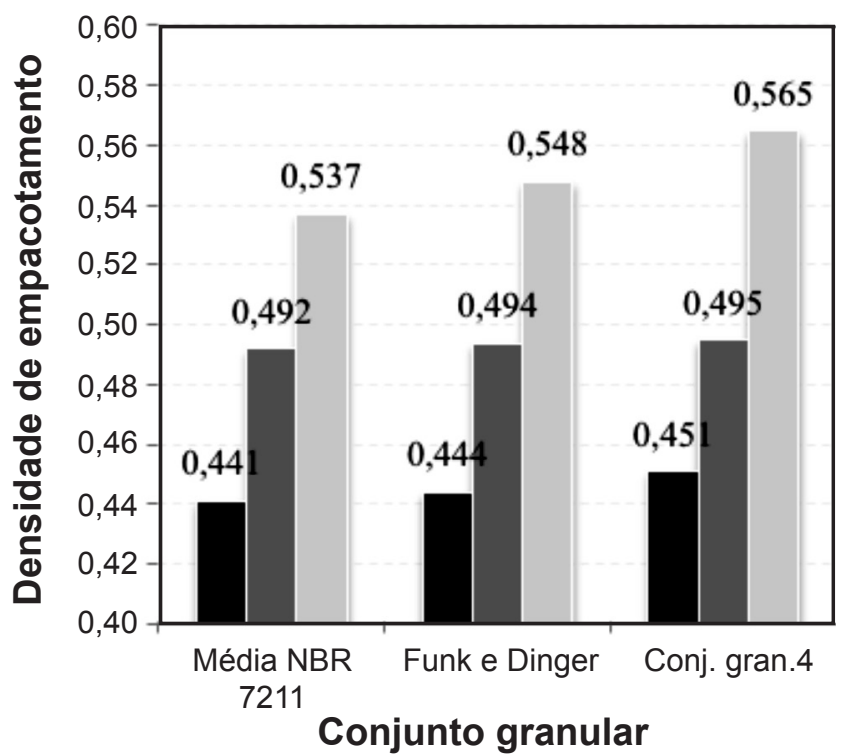

\section{- Dens. Emp. Real $(\phi) \quad$ Dens. Emp. Experimental Dens. Emp. Virtual $(\gamma)$}

Figura 5: Verificação experimental dos resultados teóricos encontrados pelo uso do modelo CPM [16].

[Figure 5: Experimental validation of the theoretical results obtained from the CPM model [16].]
$11,3 \%$ e $9,7 \%$ superior à densidade de empacotamento real teórica, para os casos da curva média da norma brasileira, da curva do modelo Funk-Dinger [14] e do conjunto granular 4, respectivamente. É natural que uma pequena diferença entre os resultados experimentais e teóricos sejam encontrados, pois os modelos são elaborados a partir de simplificações e de dados experimentais de um determinado conjunto de materiais. Ao se usar outros materiais nas campanhas experimentais, então, diferenças são observadas. Ressaltase, novamente, que não é adequada a comparação dos dados experimentais com os dados teóricos de densidade de empacotamento virtual, pois esta última não considera a compactação aplicada ao conjunto. Em realidade, a densidade de empacotamento virtual considera que cada uma das partículas foi posicionada uma a uma, de modo que o preenchimento do espaço é mais efetivo. Por isso, seu valor é superior aos resultados experimentais e à densidade de empacotamento real. Entretanto, essa condição não representa a realidade das misturas granulares.

Comparações similares à apresentada neste estudo, entre resultados experimentais e teóricos (densidade de empacotamento real), são encontradas na literatura [13, 16] para 2 classes de grãos. Em todos os casos avaliados pelos autores, a densidade de empacotamento experimental se demonstrou maior que os valores obtidos analiticamente, com variações na ordem de $2 \%$. A ordem de variação apresentada neste trabalho foi superior, podendo-se justificar o fato pelo uso de materiais com características diferentes das usadas nos demais trabalhos. De qualquer modo, ambos os trabalhos citados relatam que mesmo com as divergências, o modelo CPM [16] é passível de utilização com confiabilidade. Acrescenta-se ainda que este modelo, dentre todos os outros modelos de empacotamento de partículas, é o mais preciso, pois considera o processo de compactação e apresenta bons resultados tanto para grãos arredondados como para grãos angulares, e para condições no estado seco ou úmido [13].

Finalmente, observou-se que as densidades de empacotamento experimentais foram muito próximas umas das outras. Estes valores foram iguais a 0,492, 0,494 e 0,495 para os casos da curva média da norma brasileira, da curva do modelo Funk-Dinger [14] e do conjunto granular 4 , respectivamente. Isto significou que as densidades de empacotamento experimentais obtidas pelo modelo FunkDinger [14] e pelo conjunto granular 4 foram apenas $0,5 \%$ e $0,6 \%$ superiores à obtida pela curva média da norma brasileira. Em suma, a diferença entre os resultados experimentais foi menor que a observada entre os resultados teóricos de densidade de empacotamento real.

\section{CONCLUSÕES}

Após a realização do programa experimental proposto e da análise dos resultados obtidos, as seguintes conclusões podem ser delineadas. (i) As curvas granulométricas obtidas pelos modelos de Füller-Thompson e Andreasen-Andersen diferiram muito da realidade de conjuntos granulares, pois 
admitem partículas infinitamente pequenas sem considerar a existência de um diâmetro mínimo, o que não permite se obter uma soma de $100 \%$ das porcentagens retidas. Para viabilizar a aplicação do modelo CPM [16] para o cálculo da densidade de empacotamento, foi realizada a normalização dos valores das frações de ambas as curvas. (ii) A curva normalizada de Füller e Thompson foi a que mais se aproximou da curva de referência da norma NBR 7211 [15]. Já a curva proveniente do modelo de Funk e Dinger [14] localizou-se fora da zona ótima recomendada pela NBR 7211 [15], estando na zona utilizável inferior. Esta curva apresentou a máxima densidade de empacotamento, dentre os modelos estudados. Suas densidades de empacotamento real e experimental foram apenas $0,7 \%$ e $0,5 \%$ superiores à da curva da norma brasileira, respectivamente. (iii) A curva granulométrica referente ao conjunto granular 4, com 40\% do material retido na fração $1,2 \mathrm{~mm}$ e $20 \%$ em cada uma das demais frações, apresentou densidade de empacotamento máxima, dentre todos os conjuntos granulares estudados, estando localizada na zona utilizável inferior apontada pela NBR 7211 [15]. A distribuição igualitária de material nas frações mais finas, associada a uma maior porcentagem na fração 1,2 mm (que apresentou maior densidade de empacotamento, dentre as frações estudadas), colaborou para um melhor preenchimento de espaços. Suas densidades de empacotamento real e experimental foram apenas $2,2 \%$ e $0,6 \%$ superiores à da curva da norma brasileira, respectivamente. (iv) A curva granulométrica média da zona ótima estabelecida pela NBR 7211 [15] obteve a menor densidade de empacotamento, dentre todos os conjuntos granulares estudados. Observou-se que, no presente estudo, as curvas granulométricas localizadas na zona utilizável inferior, apontada pela mesma norma, obtiveram densidades de empacotamento levemente superior à curva localizada na zona ótima. De qualquer forma, a recomendação da zona ótima dada pela norma brasileira considera a existência de 6 classes de grãos para a areia, as quais não foram todas consideradas neste estudo, pois a areia normal do IPT apresenta somente 4 frações. Aponta-se, então, a necessidade de estudos futuros que relacionem a quantidade de classes com a densidade de empacotamento. (v) Os resultados de densidade de empacotamento experimental foram $11,6 \%, 11,3 \%$ e 9,7\% superiores à densidade de empacotamento real teórica, para os casos da curva média da norma brasileira, da curva do modelo Funk e Dinger [14] e do conjunto granular 4, respectivamente. As diferenças foram consideradas aceitáveis, devido às restrições do modelo e ao fato de este ter sido criado a partir de dados experimentais de um determinado conjunto de materiais. Ao se usar outros materiais na campanha experimental diferenças são naturalmente observadas. (vi) As densidades de empacotamento experimentais obtidas pelo modelo Funk e Dinger [14] e pelo conjunto granular 4 foram apenas $0,5 \%$ e $0,6 \%$ superiores à obtida pela curva média da norma brasileira. A diferença entre os resultados teóricos foi superior, indicando que as densidades de empacotamento reais obtidas pelo modelo Funk e Dinger [14] e pelo conjunto granular 4 foram $0,7 \%$ e 2,2\% superiores à obtida pela curva média da norma brasileira.

\section{AGRADECIMENTOS}

Os autores agradecem à empresa Concrebras, que forneceu o material, os equipamentos e o espaço de laboratório, necessários à realização dos experimentos apresentados neste trabalho.

\section{APÊNDICE: ROTEIRO DE CÁLCULO DO MODELO CPM}

A fim de propor uma melhor compreensão do procedimento de cálculo referente ao modelo CPM [16], apresenta-se um roteiro com o desenvolvimento deste. Neste trabalho, o modelo foi aplicado para obtenção da densidade de empacotamento de uma mistura com as quatro classes de tamanho de grãos disponíveis para a areia normal do IPT. Logo, o número de classes ( $n$ ) é igual a 4. Como dados de entrada para a aplicação do modelo têm-se: i) diâmetro de cada classe de tamanho de grãos, chamado de $d_{i}$; ii) volume relativo de grãos presente em cada classe, chamado de $y_{i}$; iii) densidade de empacotamento de cada classe, individualmente, chamada $\beta_{i}$.

Os diâmetros das quatro classes estudadas foram os mesmos referentes a cada fração da areia normal do IPT. Para determinar os volumes relativos de cada classe, é utilizado como exemplo a composição aleatória do conjunto granular 4 , tendo sido esta a que apresentou a maior densidade de empacotamento, dentre todos os conjuntos estudados.

Tabela A.I - Dados de entrada para a aplicação do modelo CPM.

[Table A.I - Input data needed for the CPM model.]

\begin{tabular}{ccccc}
\hline $\begin{array}{c}\text { Classe } \\
(n=4)\end{array}$ & $\mathrm{d}_{\mathrm{i}}(\mathrm{mm})$ & $\begin{array}{c}\text { Volume de cada fração para } \\
\text { o conjunto granular 4 (\%) }\end{array}$ & $\mathrm{y}_{\mathrm{i}}$ & $\beta_{\mathrm{i}}$ \\
\hline 1 & 1,20 & 40,0 & $y_{1}=40,0 / 100=0,40$ & 0,441 \\
2 & 0,60 & 20,0 & $y_{1}=20,0 / 100=0,20$ & 0,430 \\
3 & 0,30 & 20,0 & $y_{1}=20,0 / 100=0,20$ & 0,424 \\
4 & 0,15 & 20,0 & $y_{1}=20,0 / 100=0,20$ & 0,420 \\
\hline$l^{\circ}$ passo: Determinac̃óno dos efeitos de interação entre partículas
\end{tabular}

$1^{\circ}$ passo: Determinação dos efeitos de interação entre partículas 
Por fim, as densidades de empacotamento de cada classe, individualmente, foram determinadas experimentalmente segundo a Equação $\mathrm{H}$, que tem como base as massas específica e unitária dos grãos. Dessa forma, os dados de entrada necessários à aplicação do modelo, relativos a cada uma das 4 classes estudadas, estão apresentados na Tabela A.I. O procedimento de cálculo consiste em uma sequência de passos, descritos à continuação, para a obtenção dos valores da densidade de empacotamento virtual e real.

Tabela A.II - Resultados do coeficiente $\mathrm{a}_{\mathrm{ij}}$, referente ao efeito de afastamento.

[Table A.II - Results for the coefficient $a_{i j}$, referring to the loosening effect.]

\begin{tabular}{cccccc}
\hline & & \multicolumn{4}{c}{$\mathrm{j}$} \\
\multicolumn{1}{c}{$\mathrm{a}_{\mathrm{ij}}$} & 1 & 2 & 3 & 4 \\
\hline \multirow{4}{*}{$\mathrm{i}$} & 1 & 1,00 & 0,712 & 0,504 & 0,357 \\
& 2 & Valor $\nexists \mathrm{R}$ & 1,00 & 0,712 & 0,504 \\
& 3 & Valor $\not \nexists \mathrm{R}$ & Valor $\not \mathrm{R}$ & 1,00 & 0,712 \\
& 4 & Valor $\not \nexists \mathrm{R}$ & Valor $\not \nexists \mathrm{R}$ & Valor $\not \mathrm{R}$ & 1,00 \\
\hline
\end{tabular}

Tabela A.III - Resultados do coeficiente $b_{i j}$, referente ao efeito parede.

[Table A.III - Results for the coefficient $b_{i j}$, referring to the wall effect.]

\begin{tabular}{cccccc}
\hline \multicolumn{2}{c}{$\mathrm{b}_{\mathrm{ij}}$} & \multicolumn{5}{c}{$\mathrm{j}$} \\
& & 1 & 2 & 3 & 4 \\
\hline & 1 & 1,00 & Valor $\not \mathrm{R}$ & Valor $\not \mathrm{R}$ & Valor $\not \mathrm{R}$ \\
$\mathrm{i}$ & 2 & 0,646 & 1,00 & Valor $\not \mathrm{R}$ & Valor $\nexists \mathrm{R}$ \\
& 3 & 0,350 & 0,646 & 1,00 & Valor $\not \mathrm{R}$ \\
& 4 & 0,182 & 0,350 & 0,646 & 1,00 \\
\hline
\end{tabular}

Os efeitos de interação ente partículas são calculados através dos coeficientes $a_{i j}$ e $b_{i j}$ correspondentes ao efeito de afastamento e efeito parede, respectivamente, conforme Equações C e D. É importante ressaltar que é necessário calcular estes coeficientes para todas as possíveis combinações entre classes de grãos, o que resulta nas matrizes apresentadas nas Tabelas A.II e A.III.

De forma mais detalhada, para o cálculo dos coeficientes $a_{11}, a_{12}$ e $a_{2 l}$, por exemplo, têm-se os resultados apresentados nas Equações A.1 a A.3, os quais estão de acordo com os apresentados na Tabela A.II.

$a_{11}=\sqrt{1-\left(1-d_{1} / d_{1}\right)^{1,02}}=\sqrt{1-(1-1200 / 1200)^{1,02}}=1,00$

$\mathrm{a}_{12}=\sqrt{1-\left(1-\mathrm{d}_{2} / \mathrm{d}_{1}\right)^{1,02}}=\sqrt{1-(1-600 / 1200)^{1,02}}=0,712$

$\mathrm{a}_{21}=\sqrt{1-\left(1-\mathrm{d}_{1} / \mathrm{d}_{2}\right)^{1,02}}=\sqrt{1-(1-1200 / 600)^{1,02}}=\not \nexists \mathrm{R}$

Similarmente, para o cálculo dos coeficientes $b_{11}, b_{12}$ e $b_{2 l}$, por exemplo, têm-se os resultados apresentados nas Equações A.4 a A.6, os quais estão de acordo com os apresentados na Tabela A.III. O cálculo dos coeficientes $a_{i j}$ e $b_{i j}$, referentes ao efeito de afastamento e efeito parede, respectivamente, devem ser feitos para todas as possíveis combinações entre classes de grãos seguindo os mesmos princípios indicados nas Equações A.1 a A.6.

$\mathrm{b}_{11}=1-\left(1-\frac{\mathrm{d}_{1}}{\mathrm{~d}_{2}}\right)^{1,50}=1-\left(1-\frac{1200}{1200}\right)^{1,50}=1,00$

$\mathrm{b}_{12}=1-\left(1-\frac{\mathrm{d}_{1}}{\mathrm{~d}_{2}}\right)^{1,50}=1-\left(1-\frac{1200}{600}\right)^{1,50}=\not \nexists \mathrm{R}$

$\mathrm{b}_{21}=1-\left(1-\frac{\mathrm{d}_{2}}{\mathrm{~d}_{1}}\right)^{1,50}=1-\left(1-\frac{600}{1200}\right)^{1,50}=0,646$

Através das Tabelas A.II e A.III, pode-se observar que quando são combinadas classes cujos diâmetros dos grãos são iguais, ou seja, quando $d_{i}=d_{i}$, o resultado dos coeficientes é igual à unidade. Isso faz com que a diagonal principal de ambas as matrizes $a_{i j}$ e $b_{i j}$ corresponda a 1,00 . Isso se justifica, pois grãos de mesmo tamanho não provocam efeito de afastamento ou efeito parede uns sobre os outros. Estes efeitos estão presentes apenas quando os diâmetros entre as classes de grãos são diferentes. Os dados mostram, também, que os resultados encontrados para algumas combinações de classes não pertencem ao conjunto dos números reais $(\mathrm{R})$, o que impossibilita o prosseguimento do cálculo para as respectivas combinações. Para o coeficiente $a_{i j}$, isso ocorre quando o $d_{i}<d_{j}$, o que se justifica, pois o efeito de afastamento é resultado da ação de partículas de menor tamanho sobre partículas de maior tamanho, como mostrado na Fig. 1. Logo, sempre que $d_{i}<d_{j}$, não se verifica ocorrência de efeito de afastamento. Para o coeficiente $b_{i j}$ observa-se o contrário e o fato verifica-se quando $d_{i}>d_{j}$. O efeito parede é o resultado da ação de partículas de maior tamanho sobre partículas de menor tamanho, $\log$, se $d_{i}>d_{j}$, não há efeito parede. Finalmente, nota-se que os resultados apresentados nas diagonais secundárias das matrizes dadas nas Tabelas A.II e A.III são iguais. Isto ocorre sempre que a relação entre os diâmetros das classes estudadas for constante, o que facilita a conferência dos resultados encontrados em todas as células das matrizes. No presente estudo, a razão entre os diâmetros das classes é constante, igual a 2 .

\section{$2^{\circ}$ passo: Determinação da densidade de empacotamento virtual}

O segundo passo consiste em determinar a densidade de empacotamento virtual através da Equação E, considerando em cada etapa de cálculo uma classe de grãos como dominante $(i)$. Dessa forma, foram desenvolvidas quatro equações, uma para cada classe dominante conforme indicado nas Equações A.7 a A.14:

Substituindo os valores correspondentes nas Equações A.8, A.10, A.12 e A.14 têm-se, respectivamente, as equações 


$$
\begin{aligned}
& \gamma_{1}=\frac{\beta_{1}}{1-\sum_{j=1}^{1-1=0}\left[1-\beta_{1}+b_{1 j} \cdot \beta_{1}\left(1-\frac{1}{\beta_{j}}\right)\right] \cdot y_{j}-\sum_{j=1+1=2}^{4}\left[1-a_{1 j} \cdot \frac{\beta_{1}}{\beta_{j}} \cdot \gamma_{j}\right.} \\
& \gamma_{1}=\frac{\beta_{1}}{1-\left\{\left[1-a_{12} \cdot \frac{\beta_{1}}{\beta_{2}}\right] \cdot \gamma_{2}+\left[1-a_{13} \cdot \frac{\beta_{1}}{\beta_{3}}\right] \cdot \gamma_{3}+\left[1-a_{14} \cdot \frac{\beta_{1}}{\beta_{4}}\right] \cdot \gamma_{4}\right\}} \\
& \gamma_{2}=\frac{\beta_{2}}{1-\sum_{j=1}^{2-1=1}\left[1-\beta_{2}+b_{2 j} \cdot \beta_{2}\left(1-\frac{1}{\beta_{j}}\right)\right] \cdot y_{j}-\sum_{j=2+1=3}^{4}\left[1-a_{2 j} \cdot \frac{\beta_{2}}{\beta_{j}} \cdot \gamma_{j}\right.} \\
& \gamma_{3}=\frac{\beta_{2}}{1-\left[1-\beta_{2}+b_{21} \cdot \beta_{2}\left(1-\frac{1}{\beta_{1}}\right)\right] \cdot y_{1}-\left\{\left[1-a_{23} \cdot \frac{\beta_{2}}{\beta_{3}}\right] \cdot y_{3}+\left[1-a_{24} \cdot \frac{\beta_{2}}{\beta_{4}}\right] \cdot \gamma_{4}\right\}} \\
& \gamma_{3}=\frac{\beta_{3}}{\left.1-\left\{\left[1-\beta_{3}+b_{31} \cdot \beta_{3}\left(1-\frac{1}{\beta_{1}}\right)\right] \cdot y_{1}+\left[1-\beta_{3}+b_{32} \cdot \beta_{3}\left(1-\frac{1}{\beta_{2}}\right)\right] \cdot y_{2}\right\}+\left[1-\beta_{3}+b_{3 j} \cdot \beta_{3}\left(1-\frac{1}{\beta_{j}}\right)\right] \cdot y_{j}-\sum_{j=3+1=4}^{4} \cdot \frac{\beta_{3}}{\beta_{4}}\right] \cdot y_{4}} \\
& \gamma_{4}=\frac{\beta_{3}\left[-a_{3 j} \cdot \frac{\beta_{3}}{\beta_{j}}\right] \cdot \gamma_{j}}{1-\sum_{j=1}^{4-1=3}\left[1-\beta_{4}+b_{4 j} \cdot \beta_{4}\left(1-\frac{1}{\beta_{j}}\right)\right] \cdot y_{j}-\sum_{j=4+1=5}^{4}\left[1-a_{4 j} \cdot \frac{\beta_{4}}{\beta_{j}}\right] \cdot \gamma_{j}}
\end{aligned}
$$

A.15 a A.18, que apresentam os resultados de densidade de empacotamento virtual, considerando cada uma das classes como dominantes:
Com base nos resultados desenvolvidos tem-se a densidade de empacotamento virtual do conjunto, a qual corresponde ao menor valor encontrado, segundo a Equação

$$
\begin{aligned}
& \gamma_{1}=\frac{0,441}{1-\left\{\left[1-1,712 \cdot \frac{0,441}{0,430}\right] \cdot 0,20+\left[1-0,504 \cdot \frac{0,441}{0,424}\right] \cdot 0,20+\left[1-0,357 \cdot \frac{0,441}{0,420}\right] \cdot 0,20\right\}}=0,608 \\
& \gamma_{2}=\frac{0,431}{1-\left[1-0,431+0,646 \cdot 0,431\left(1-\frac{1}{0,441}\right)\right] \cdot 0,40-\left\{\left[1-0,712 \cdot \frac{0,431}{0,441}\right] \cdot 0,2+\left[1-0,504 \cdot \frac{0,431}{0,420}\right] \cdot 0,2\right\}}=0,565
\end{aligned}
$$$$
\gamma_{3}=\frac{0,424}{1-\left\{\left[1-0,424+0,350 \cdot 0,424\left(1-\frac{1}{0,441}\right)\right] \cdot 0,40+\left[1-0,424+0,646 \cdot 0,424\left(1-\frac{1}{0,431}\right)\right] \cdot 0,20\right\}-\left[1-0,712 \cdot \frac{0,424}{0,420}\right] \cdot 0,20}=0,569
$$ 
F e como segue na Equação A.19:

$$
\gamma=\operatorname{MIN}\left(\gamma_{\mathrm{i}}\right) 1 \leq \mathrm{i} \leq 4=0,565
$$

Observa-se que a densidade de empacotamento virtual do conjunto granular foi obtida ao considerar a classe 2 , de tamanho $0,6 \mathrm{~mm}$, como dominante $\left(\gamma_{2}=0,565\right)$.

\section{$3^{\circ}$ passo: Determinação da densidade de empacotamento real}

A última etapa de cálculo consiste em determinar a densidade de empacotamento real $(\phi)$, a qual considera a aplicação de uma energia de compactação $(K)$ relacionada ao processo experimental utilizado na determinação dos valores de $\beta_{\mathrm{i}}$. Nesse caso adotou-se um valor de $K=4,5$, pois este corresponde ao processo de aplicação de compactação por apiloamento, sendo este o processo utilizado experimentalmente (método A descrito na norma NBR NM 45 [22]). Assim, a densidade de empacotamento real do conjunto pode ser determinada indiretamente segundo a Equação G. Ao desenvolver a referida equação e substituir as incógnitas pelos valores obtêm-se as Equações A.20 e A.21:

$\mathrm{K}=\sum_{\mathrm{i}=1}^{4} \frac{\mathrm{y}_{\mathrm{i}} / \beta_{\mathrm{i}}}{1 / \phi-1 / \mathrm{y}_{\mathrm{i}}}=\frac{\mathrm{y}_{1} / \beta_{1}}{1 / \phi-1 / \mathrm{y}_{1}}=\frac{\mathrm{y}_{2} / \beta_{2}}{1 / \phi-1 / \mathrm{y}_{2}}=\frac{\mathrm{y}_{3} / \beta_{3}}{1 / \phi-1 / \mathrm{y}_{3}}=\frac{\mathrm{y}_{4} / \beta_{4}}{1 / \phi-1 / \mathrm{y}_{4}}$

$4,5=\frac{0,40 / 0,441}{1 / \phi-1 / 0,608}+\frac{0,20 / 0,430}{1 / \phi-1 / 0,565}+\frac{0,20 / 0,424}{1 / \phi-1 / 0,569}+\frac{0,20 / 0,420}{1 / \phi-1 / 0,611}$

A Equação A.21 resulta em uma equação do $4^{\circ}$ grau, por apresentar 4 termos referentes às 4 classes de grãos do presente estudo. Dessa forma, sua resolução foi facilitada pela utilização do software matemático Maple (http://www. maplesoft.com/products/Maple/) o qual gera o resultado das quatro raízes da equação. A densidade de empacotamento real $(\phi)$ corresponde à raiz $\ni \mathrm{R}$ de menor valor, nesse caso equivalente a 0,451 .

\section{REFERÊNCIAS}

[1] E. Gartner, H. Hirao, Cem. Concr. Res. 78 (2015) 126.

[2] M.L. Berndt, Renew. Energ. 83 (2015) 608.

[3] T.C. Powers, The properties of fresh concrete, John Wiley \& Sons, New York (1968).

[4] V. Wong, K.W. Chan, A.K.H. Kwan, Org. Technol.
Manag. Constr. 5 (2013) 844.

[5] J.C. Benezet, P. Adamiec, M. Nemoz-Gaillard, Study of real granular assemblies, Centre dês Matériaux de Grande Diffusion, Ecoledes Mines d'Alès, France (2006).

[6] H.C. Wong, A.K.H. Kwan, Mater. Struct. 41 (2008) 689. [7] A.L. Castro, V.C. Pandolfelli, Cerâmica 55 (2009) 18.

[8] W.W.S. Fung, A.K.H. Kwan, H.H.C. Wong, Mater. Struct. 42 (2009) 631.

[9] A.K.H. Kwan, C.F. Mora, Magazine Concr. Res. 53 (2001) 91.

[10] M. Alexander, S. Mindess, Aggregates in concrete, Taylor \& Francis, New York (2005).

[11] A.K.H. Kwan, K.W. Chan, V. Wong, Powder Technol. 237 (2013) 172.

[12] W.X. Xu, H.S. Chen, Phisica A 392 (2012) 416.

[13] S.A.A.M. Fennis, "Design of ecological concrete by particle packing optimization", Tese Dr., TU Delft, Amersfoort, Holanda (2011).

[14] J.E. Funk, D.R. Dinger, Caol grinding and particle size distribution studies for coal-water slurries at high solids content, Empire State Electric Energy Research Corporation, New York (1980).

[15] ABNT - NBR 7211, Associação Brasileira de Normas Técnicas, "Agregados para concreto, Especificação" (2009).

[16] F. De Larrard, Concrete mixture proportioning: a scientific approach, E \& FN Spon, London (1999).

[17] ABNT - NBR 7214, Associação Brasileira de Normas Técnicas, "Areia normal para ensaio de cimento" (1982).

[18] W. Toufar, M. Born, E. Klose, Freiberger Forschungsheft A 558 (1979) 29.

[19] J.D. Dewar, Computer modelling of concrete mixtures, E \& FN Spon, London (1999).

[20] F. Schwanda, Zement Beton 37 (1966) 8.

[21] A.B. Yu, N. Standish, Ind. Eng. Chem. Res. 30 (1991) 1372 .

[22] ABNT - NBR NM 45, Associação Brasileira de Normas Técnicas, Agregados, "Determinação da massa unitária e do volume de vazios" (2006).

[23] ABNT - NBR NM 53, Associação Brasileira de Normas Técnicas, "Agregado graúdo, Determinação da massa específica e massa específica aparente” (2009).

[24] A.B. Yu, C.L. Feng, R.Y. Yang, Powder Technol. 130 (2003) 70 .

[25] T.C. Hales, Ann 691 Math 162 (2005) 1065.

[26] A.P. Silva, A.M. Segadães, T.C. Deezas, Anais do $47^{\circ}$ Congresso Brasileiro de Cerâmica, João Pessoa, PB (2003). (Rec. 20/11/2015, Rev. 02/02/2016, 21/03/2016, Ac. 24/05/2016) 